\title{
Interplay between Vortex Dynamics and Superconducting Gap Structure in Layered Organic Superconductors
}

\author{
Syuma Yasuzuka
}

Citation: Yasuzuka, S. Interplay between Vortex Dynamics and Superconducting Gap Structure in Layered Organic Superconductors. Crystals 2021, 11, 600. https:// doi.org/10.3390/cryst11060600

Academic Editor: Toshio Naito

Received: 24 March 2021

Accepted: 20 May 2021

Published: 26 May 2021

Publisher's Note: MDPI stays neutral with regard to jurisdictional claims in published maps and institutional affiliations.

Copyright: (C) 2021 by the author. Licensee MDPI, Basel, Switzerland. This article is an open access article distributed under the terms and conditions of the Creative Commons Attribution (CC BY) license (https:// creativecommons.org/licenses/by/ $4.0 /)$.
Research Center for Condensed Matter Physics, Hiroshima Institute of Technology, Hiroshima 731-5193, Japan; yasuzuka@cc.it-hiroshima.ac.jp; Tel.: +81-82-921-6930

\begin{abstract}
Layered organic superconductors motivate intense investigations because they provide various unexpected issues associated with their low dimensionality and the strong electron correlation. Since layered organic superconductors possess simple Fermi surface geometry and they often share similarities to the high temperature oxide superconductors and heavy fermion compounds, research on layered organic superconductors is suitable for understanding the essence and nature of strongly correlated electron systems. In strongly correlated electron systems, one of the central problems concerning the superconducting (SC) state is the symmetry of the SC gap, which is closely related to the paring mechanism. Thus, experimental determination of the SC gap structure is of essential importance. In this review, we present the experimental results for the in-plane angular variation of the flux-flow resistance in layered organic superconductors $\mathrm{K}-(\mathrm{ET})_{2} \mathrm{Cu}(\mathrm{NCS})_{2}, \beta^{\prime \prime}-(\mathrm{ET})_{2} \mathrm{SF}_{5} \mathrm{CH}_{2} \mathrm{CF}_{2} \mathrm{SO}_{3}$, and $\lambda$-(BETS $)_{2} \mathrm{GaCl}_{4}$. The interplay between the vortex dynamics and nodal structures is discussed for these superconductors.
\end{abstract}

Keywords: layered organic conductor; unconventional superconductivity; vortex dynamics; $d$-wave pairing symmetry; superconducting gap structure; magnetic field; flux-flow resistivity

\section{Introduction}

Physics of organic conductors has provided the fascinating and rich issues associated with the low dimensionality and effect of electron correlation [1]. One of the most interesting systems are the layered organic conductors composed of the ET donor molecule, where ET denotes bis(ethylenedithio)tetrathiafulvalene. The ET donor molecules form two-dimensional (2D) conducting layers that are separated by insulating anion layers with a monovalent ion $\mathrm{X}^{-}$. Figure 1 a shows schematic structure of ET (or BETS) donor conducting layers and anion insulating layers in a layered organic conductor, where BETS denotes bis(ethylenedithio)tetraselenafulvalene. The molecular structures of ET and BETS are shown in the upper right side of Figure 1a. In layered organic superconductors, there are several types (labeled by Greek letters) in packed ET donor layers. The K-, $\beta^{\prime \prime}-$, and $\lambda$-type arrangements in donor layers are shown in Figure $1 \mathrm{~b}-\mathrm{d}$. In the $\mathrm{K}$ type compounds, $\mathrm{K}-(\mathrm{ET})_{2} X$, the measurements of Shubnikov-de Haas (SdH) and de Haas-van Alphen (dHvA) oscillations have elucidated the presence of a well-defined Fermi surface (FS) with simple structures [2]. Moreover. the moderately heavy effective mass revealed by $\mathrm{SdH}$ and $\mathrm{dHvA}$ experiments suggests that electron correlation plays a significant role on determining the physical properties of the normal state as well as superconducting (SC) state. It was suggested that superconductivity appears in proximity to the antiferromagnetic insulating state in the electronic phase diagram [3,4]. Since some of these unusual physical properties suggest similarities with high- $T_{\mathrm{c}}$ cuprates, many researchers have pointed out that the spin fluctuations play a vital role for the appearance of SC state [5-8]. 


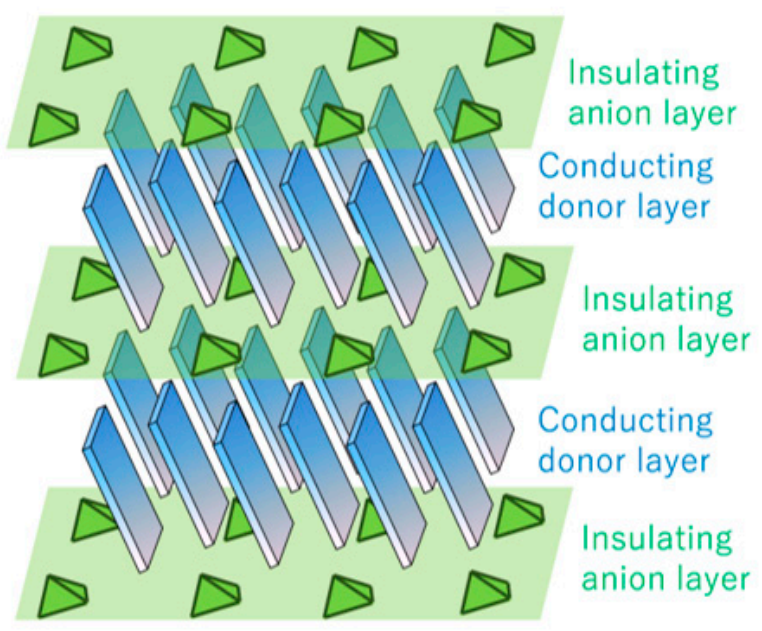

(a) $\beta^{\prime \prime}-(\mathrm{ET})_{2} \mathrm{SF}_{5} \mathrm{CH}_{2} \mathrm{CF}_{2} \mathrm{SO}_{3}$

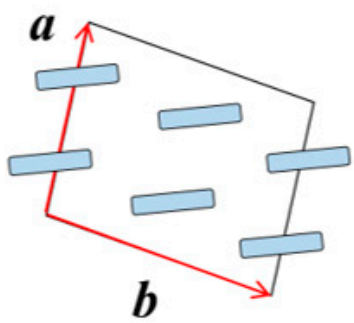

( $\beta$ " -type arrangement)

(c)

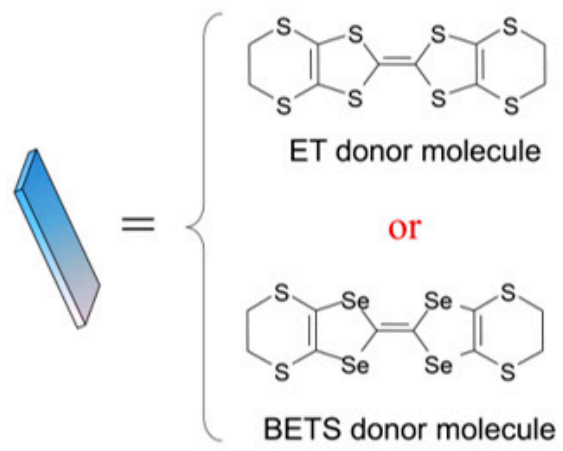

$\lambda-(\mathrm{BETS})_{2} \mathrm{GaCl}_{4}$

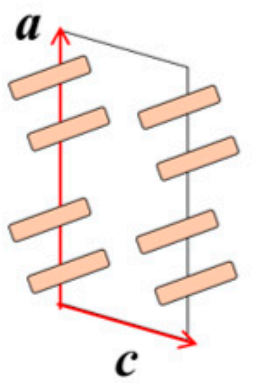

( $\lambda$-type arrangement)

(d)

Figure 1. (a) (Upper left side) Schematic structure of donor conducting layers and anion insulating layers in a layered organic conductor. (Upper right side) Structures of ET and BETS donor molecules. (Lower side) Three patterns within packed donor conducting layers for (b) K-, (c) $\beta^{\prime \prime}-$, and (d) $\lambda$-type arrangements.

For layered organic superconductors, many experimental studies have pointed out the existence of nodes in the SC gap [9-15]. The SC gap structure in unconventional superconductors mostly have nodes parallel to certain orientations in the momentum space. The confirmation of point or line nodes is often discussed from power-law temperature $(T)$ dependences in physical quantities such as the heat capacity or the nuclear spin relaxation rate. However, determination of the SC gap structure is a harder task. For example, the SC gap symmetry of $d_{x y}$ (with nodes along vertical and horizontal directions) differs from $d_{x}^{2}-y^{2}$ (diagonal direction) only in the location of the line nodes ( $\pi / 4$ rotation of the latter becomes identical with the former). Therefore, direction-sensitive experimental methods are highly needed to distinguish between these gap structures. Since the SC gap structure is intimately associated with the pairing mechanism [16], it is of fundamental importance to elucidate the SC gap structure.

In the vortex state of $d$-wave superconductors, gapless quasiparticles (QPs) extending outside the vortex core play an important role for determining their physical properties at magnetic fields $[17,18]$. As an effect of applied magnetic field on $d$-wave superconductors, Volovik [17] theoretically introduced a "Doppler shifted state" in the energy spectrum of the QP which is induced by superfluid flow around the vortices. The QPs with low energy are induced by the application of magnetic field, mainly around the nodes where the QP velocity is perpendicular to the magnetic field orientation. The QP density depends on 
the relative direction of the magnetic field with respect to the nodes. Derived from the Doppler sifted state that depend on the magnetic field orientation, the dependence of the heat capacity and the thermal conductivity on applied field orientation was demonstrated to be a useful probe for detecting the nodal directions in a SC gap structure $[14,15,19]$.

Up to now, we have focused on the interplay between vortex dynamics and the SC gap structure in layered organic superconductors because the flux-flow resistivity is a measure of QP dissipation in the vortex dynamics [20-24]. To investigate the correlation between vortex dynamics and the SC gap structure, we measured the in-plane anisotropy of the flux-flow resistivity for the layered organic superconductors $\mathrm{K}-(\mathrm{ET})_{2} \mathrm{Cu}(\mathrm{NCS})_{2}$, $\beta^{\prime \prime}-(\mathrm{ET})_{2} \mathrm{SF}_{5} \mathrm{CH}_{2} \mathrm{CF}_{2} \mathrm{SO}_{3}$, and $\lambda-(\mathrm{BETS})_{2} \mathrm{GaCl}_{4}$ [25-27]. SC parameters for these superconductors are listed in Table 1 . As shown below, we clearly observed the flux-flow resistivity with fourfold-symmetric anisotropy, owning to the $d$-wave SC gap symmetry in $\mathrm{K}-(\mathrm{ET})_{2} \mathrm{Cu}(\mathrm{NCS})_{2}$ and $\beta^{\prime \prime}-(\mathrm{ET})_{2} \mathrm{SF}_{5} \mathrm{CH}_{2} \mathrm{CF}_{2} \mathrm{SO}_{3}$. On the other hand, twofold symmetric anisotropy was found in $\lambda$-(BETS $)_{2} \mathrm{GaCl}_{4}$ although $\lambda$-(BETS) ${ }_{2} \mathrm{GaCl}_{4}$ possesses the similar FS and SC gap structures in the former two superconductors. Interplay between in-plane anisotropy of vortex dynamics and nodal SC gap structures for these superconductors are discussed below.

Table 1. Superconducting parameters for $\mathrm{K}-(\mathrm{ET})_{2} \mathrm{Cu}(\mathrm{NCS})_{2}[2], \beta^{\prime \prime}-(\mathrm{ET})_{2} \mathrm{SF}_{5} \mathrm{CH}_{2} \mathrm{CF}_{2} \mathrm{SO}_{3}[2,28-30]$ and $\lambda-(\mathrm{BETS})_{2} \mathrm{GaCl} 4$ $[2,31,32]$.

\begin{tabular}{ccccc}
\hline Properties & Unit & $\kappa-(\mathrm{ET})_{\mathbf{2}} \mathbf{C u}(\mathbf{N C S})_{\mathbf{2}}$ & $\left.\boldsymbol{\beta}^{\prime \prime-(} \mathbf{E T}\right)_{\mathbf{2}} \mathbf{S F}_{\mathbf{5}} \mathbf{C H}_{\mathbf{2}} \mathbf{C F}_{\mathbf{2}} \mathbf{S O}_{\mathbf{3}}$ & $\boldsymbol{\lambda}$ \\
\hline$T_{\mathrm{c}}$ & $\mathrm{K}$ & $8.7-10.4$ & 5.2 & $5-8$ \\
$B_{\mathrm{c} 1 \|}$ & $\mathrm{mT}$ & 0.2 & 0.006 & 5.2 \\
$B_{\mathrm{c} 1 \perp}$ & $\mathrm{mT}$ & 6.5 & 2 & 8.2 \\
$B_{\mathrm{c} 2 \|}$ & $\mathrm{T}$ & $30-35$ & 10.4 & 12 \\
$B_{\mathrm{c} 2 \perp}$ & $\mathrm{T}$ & 6 & 1.4 & 3 \\
$\lambda_{\|}$ & $\AA$ & $5100-20,000$ & $10,000-20,000$ & 1500 \\
$\lambda_{\perp}$ & $\mu \mathrm{GaCl}$ & $400-800$ & 105 \\
$\xi_{\|}$ & $\AA$ & $40-200$ & 144 & $9-14$ \\
$\xi_{\perp}$ & $\AA$ & 74 & 7.9 & 107 \\
$\kappa_{\|}$ & $\AA$ & $100-200$ & 59 & \\
\hline
\end{tabular}

\section{Experimental Methods}

Single crystals of $\mathrm{K}-(\mathrm{ET})_{2} \mathrm{Cu}(\mathrm{NCS})_{2}, \beta^{\prime \prime}-(\mathrm{ET})_{2} \mathrm{SF}_{5} \mathrm{CH}_{2} \mathrm{CF}_{2} \mathrm{SO}_{3}$, and $\lambda-(\mathrm{BETS})_{2} \mathrm{GaCl}_{4}$ were synthesized electrochemically. The interlayer resistance was measured by a fourterminal ac method, where the electric current is parallel to the least conducting direction. To investigate the in-plane angular variation of the flux-flow resistance for layered organic superconductors, it is crucial to rotate applied field parallel to the conducting layers with high accuracy because a slight field-angle misalignment gives rise to twofold anisotropy of the magnetoresistance related to the huge $H_{\mathrm{c} 2}$ anisotropy [33]. To check the reproducibility, four samples were simultaneously mounted on a two-axis rotator in a ${ }^{4} \mathrm{He}$ cryostat with a 17-T SC magnet. By using the rotator, it is possible to sweep the $\theta$ angle with a resolution of $\Delta \theta=0.1^{\circ}$, where $\theta$ is the polar angle between the least conducting axis and magnetic field direction. In addition, we can discretely control the plane of rotation, which is represented by the azimuthal angle $\phi$ with intervals of $\Delta \phi=5^{\circ}$ or 10 within the conducting layers.

To investigate the in-plane anisotropy of the flux-flow resistance at a constant magnetic field, the resistivity as a function of the angle $\theta$ at various fixed $\phi$ was measured because we can easily find the position of the $H$ parallel to the conducting plane at any $\phi$. For example, the interlayer resistivity of $\mathrm{K}-(\mathrm{ET})_{2} \mathrm{Cu}(\mathrm{NCS})_{2}$ is shown in Figure 2a [25]. For the magnetic field exactly applied parallel to the conducting plane, the $\rho(\theta)$ curve shows a sharp drop due to the SC transition at the lowest current density of $0.5 \mathrm{~mA} / \mathrm{cm}^{2}$. It is remarkable that the resistivity at $\theta=90^{\circ}$ depends strongly on the current density. A sharp peak is clearly observed at $\theta=90^{\circ}$ for highest current density of $100 \mathrm{~mA} / \mathrm{cm}^{2}$. The resistivity for $\left|\theta-90^{\circ}\right|>5^{\circ}$ is independent of current density of up to $100 \mathrm{~mA} / \mathrm{cm}^{2}$. Thus, the 
effect of Joule heating is negligibly small. Instead, the sharp peaks are due to the vortex dynamics [25,33-38]. Similar features are found for $\beta^{\prime \prime}-(\mathrm{ET})_{2} \mathrm{SF}_{5} \mathrm{CH}_{2} \mathrm{CF}_{2} \mathrm{SO}_{3}$ in Figure $2 \mathrm{~b}$ and $\lambda$-(BETS) ${ }_{2} \mathrm{GaCl}_{4}$ in Figure 2c $[26,27]$.
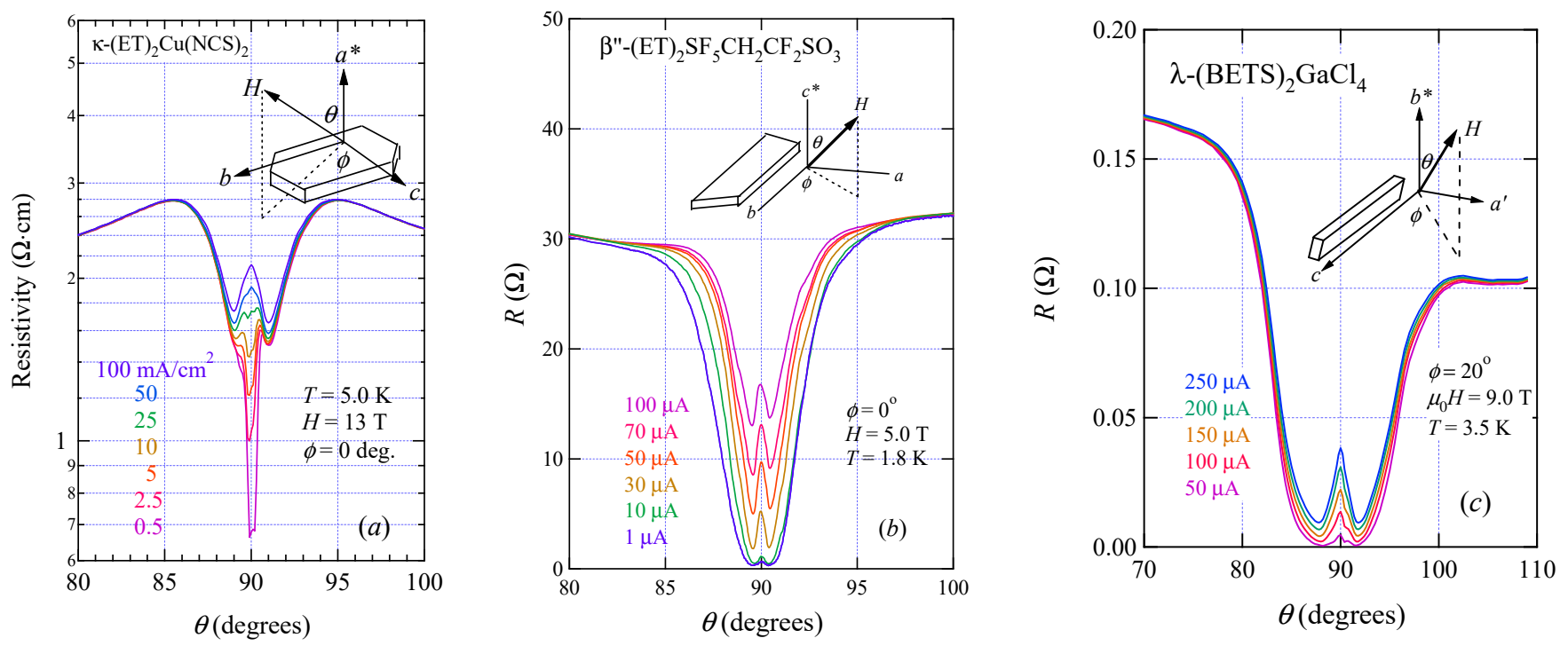

Figure 2. Interlayer resistance as a function of polar angle $\theta$ for $\kappa-(\mathrm{ET})_{2} \mathrm{Cu}(\mathrm{NCS})_{2}(\mathbf{a}), \beta^{\prime \prime}-(\mathrm{ET})_{2} \mathrm{SF}_{5} \mathrm{CH}_{2} \mathrm{SF}_{2} \mathrm{SO}_{3}(\mathbf{b})$, and $\lambda$-(BETS) ${ }_{2} \mathrm{GaCl}_{4}$ (c). The insets show the definition of $\theta$ and $\phi$. Adapted from refs. [25-27].

\section{Results and Discussion}

\section{1. $\kappa-(E T)_{2} \mathrm{Cu}(\mathrm{NCS})_{2}$}

The nature of the K-type organic superconductor K-(ET) $)_{2} X$ possesses similar features to that of high- $T_{\mathrm{c}}$ cuprates, such as quasi-2D electronic structure and the competition of its SC phase with the antiferromagnetic insulating phase [2-4]. To elucidate the pairing mechanism in the K-(ET) 2 X system, its SC gap symmetry has been extensively investigated from both experimental and theoretical points of view [9-14]. Various experiments such as NMR [9], heat capacity [10,11], and mm-wave transmission measurements [12] suggested presence of $d$-wave SC gap in K-(ET) $)_{2} \mathrm{Cu}(\mathrm{NCS})_{2}$. As for the nodal direction, scanning tunneling microscopy (STM) [13] and thermal conductivity experiments [14] suggested the line node gap rotated $45^{\circ}$ relative to the $b$ and $c$ axes $\left(d_{x}^{2}-y^{2}\right.$ symmetry). Malone et al. [15] argued in favor of $d_{x y}$ symmetry (the line nodes along the $b$ and $c$ axes) from the heat capacity measurement. These results have demonstrated the $d$-wave superconductivity in the $\mathrm{K}-(\mathrm{ET})_{2} \mathrm{X}$ system, but the location of gap nodes is still controversial. In this section, we present in-plane anisotropy of upper critical field and vortex flow resistivity for K$(\mathrm{ET})_{2} \mathrm{Cu}(\mathrm{NCS})_{2}$ and discuss the nodal structure.

\subsubsection{In-Plane Anisotropy of Upper Critical Field in K-(ET) ${ }_{2} \mathrm{Cu}(\mathrm{NCS})_{2}$}

To discuss the in-plane anisotropy of upper critical field, measurements of the interlayer resistance were performed for $\phi=0^{\circ}(H / / c), \phi=45^{\circ}, \phi=90^{\circ}(H / / b)$. Figure 3a presents the temperature dependence of interlayer resistance for various values of magnetic field applied along the $b$-axis $\left(\phi=90^{\circ}\right)$ up to $14.8 \mathrm{~T}$. Due to a broad transition into the SC state, the following four criteria are used here to extract the SC transition temperature at each magnetic field. These are shown in Figure 3a, where the four criteria of the SC transition temperature: a "junction" $T_{\mathrm{J}}$, a "midpoint" $T_{\mathrm{M}}$, "a zero resistance extrapolation" $T_{X}$ (ignoring the tail near $R=0$ ), and "zero resistance point" $T_{Z}$ are defined. A similar fashion was confirmed for $\phi=45^{\circ}$ and $\phi=0^{\circ}(H / / c)$.

Figure $2 \mathrm{~b}$ presents a $\mathrm{T}-\mathrm{H}$ phase diagram for three magnetic field directions, using the four criteria. All criteria clearly show highly isotropic behavior within our experimental error, suggesting the lack of in-plane anisotropy of $H_{\mathrm{c} 2}$ in spite of many suggestions of 
$d$-wave pairing symmetry in $\mathrm{K}-(\mathrm{ET})_{2} \mathrm{Cu}(\mathrm{NCS})_{2}$. This is consistent with previous magnetoresistance study by Nam et al. [39]. Lower temperatures will be needed for confirmation of an expected $d$-wave nature in $H_{\mathrm{c} 2}$. In addition, we find change of slope in $H_{\mathrm{c} 2}$ line near $T_{\mathrm{C}}$, suggesting the dimensional crossover from anisotropic 3D SC to Josephson coupled 2D SC state [40-42]. It means that despite short coherence length along the interlayer direction, the SC state can be regarded as anisotropic 3D SC state only near $H_{\mathrm{c} 2}$ line.
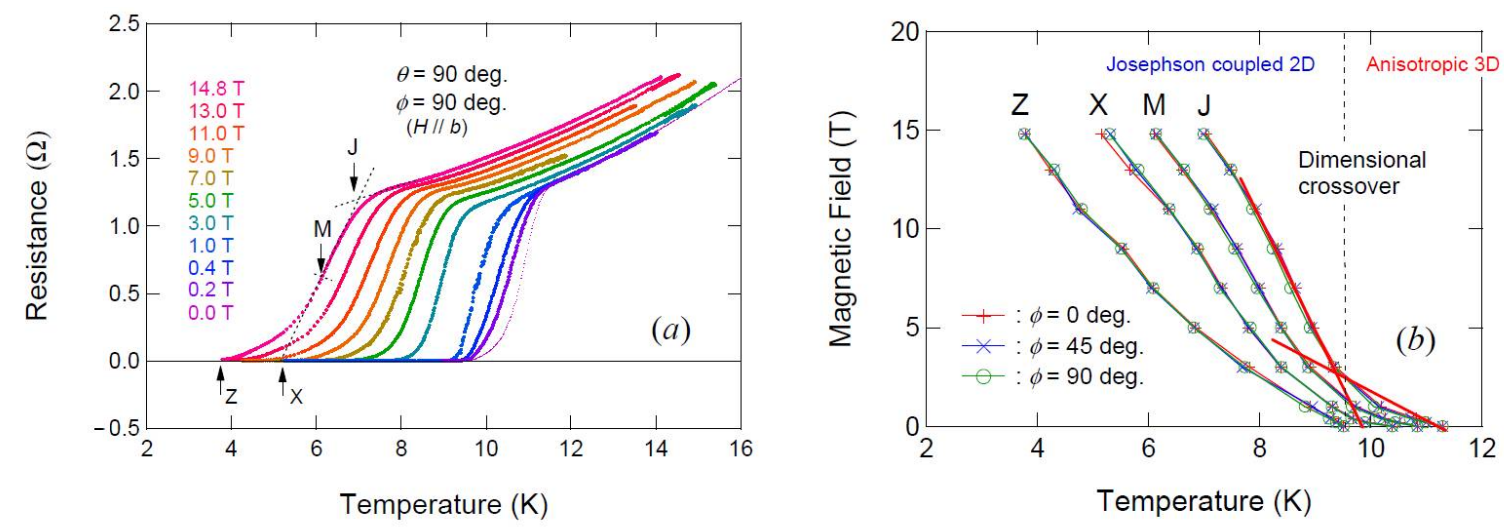

Figure 3. (a) Temperature dependence of interlayer resistance for various magnetic fields $H / / b\left(\phi=90^{\circ}\right)$ in $\mathrm{k}-$ $(\mathrm{ET})_{2} \mathrm{Cu}(\mathrm{NCS})_{2}$. (b) $H-T$ phase diagram derived from (a), where four criteria, J (junction), $\mathrm{M}$ (midpoint), $\mathrm{X}(\mathrm{R} \rightarrow 0)$, and $\mathrm{Z}(R=0)$ are plotted for $T_{\mathrm{c}}(H)$.

\subsubsection{In-Plane Anisotropy of Vortex Dynamics in K-(ET) $)_{2} \mathrm{Cu}(\mathrm{NCS})_{2}$}

Figure 4a presents the interlayer resistivity as a function of polar angle $\theta$ at various $\phi$ values with an interval of $\Delta \phi=5^{\circ}$. As seen in Figure $4 \mathrm{a}$, there is the peak structure at $\theta=90^{\circ}$ at all $\phi$ values, which confirms the vortex motion for all in-plane field directions. The peak structure gradually changes with $\phi$. To discuss the in-plane anisotropic effect in flux-flow resistance, we display the $\phi$ dependence of the interlayer resistivity at $\theta=90^{\circ}$ at various temperatures in Figure $4 \mathrm{~b}$. In the entire temperature range measured, the $\rho(\phi, T)$ curves show minima at $\phi=0^{\circ}(\mathrm{H} / / \mathrm{c})$ and $90^{\circ}(\mathrm{H} / / \mathrm{b})$. At temperatures beyond $7.8 \mathrm{~K}$, a sinusoidal angular variation was observed. At elevated temperature, the amplitude of oscillatory behavior is weakened but survives even at $18 \mathrm{~K}$, where the SC phase is vanished. At temperatures below $7.8 \mathrm{~K}$, we find a non-sinusoidal fourfold angular-variation: cusp-like minima are found at $\phi=0^{\circ}$ and $90^{\circ}$ that are independent of temperature.

As seen in Figure 4 b, the $\rho(\phi)$ curves seem to be described by a fourfold symmetric origin. Takanaka and Kuboya theoretically showed that the angular variation of the upper critical field is described as $H_{\mathrm{c} 2}(\phi) \propto \cos 4 \phi$ in the $d$-wave superconductivity [43-45]. The $\rho(\phi)$ curves are naively consistent with this theory; the maximum and minimum of $H_{\mathrm{c} 2}$ give the minimum and maximum resistivities, respectively. When $d_{x}^{2}-y^{2}$ pairing is assumed, $H_{\mathrm{c} 2}(\phi)$ has maxima at $\phi=0^{\circ}(H / / c)$ and $\phi=90^{\circ}(H / / b)$, and a minimum at $\phi=45^{\circ}$, whereas $d_{x y}$ pairing leads to $H_{\mathrm{c} 2}$ with maxima and minima reversed with respect to $d_{x}^{2}-y^{2}$ pairing. Thus, the fourfold symmetry in Figure $3 \mathrm{~b}$ is not inconsistent with the $H_{\mathrm{c} 2}$ anisotropy originating from the $d_{x}^{2}-y^{2}$ pairing state. However, detailed magnetoresistance studies at 1.5 and $4.2 \mathrm{~K}$ [39] as well as Figure 3 showed the lack of in-plane anisotropy of $H_{\mathrm{c} 2}$. In addition, the cusp-like minima that appear below $7.8 \mathrm{~K}$ cannot be represented by a simple $\cos 4 \phi$ dependence. Thus, another mechanism to describe these oscillation patterns is necessary. 

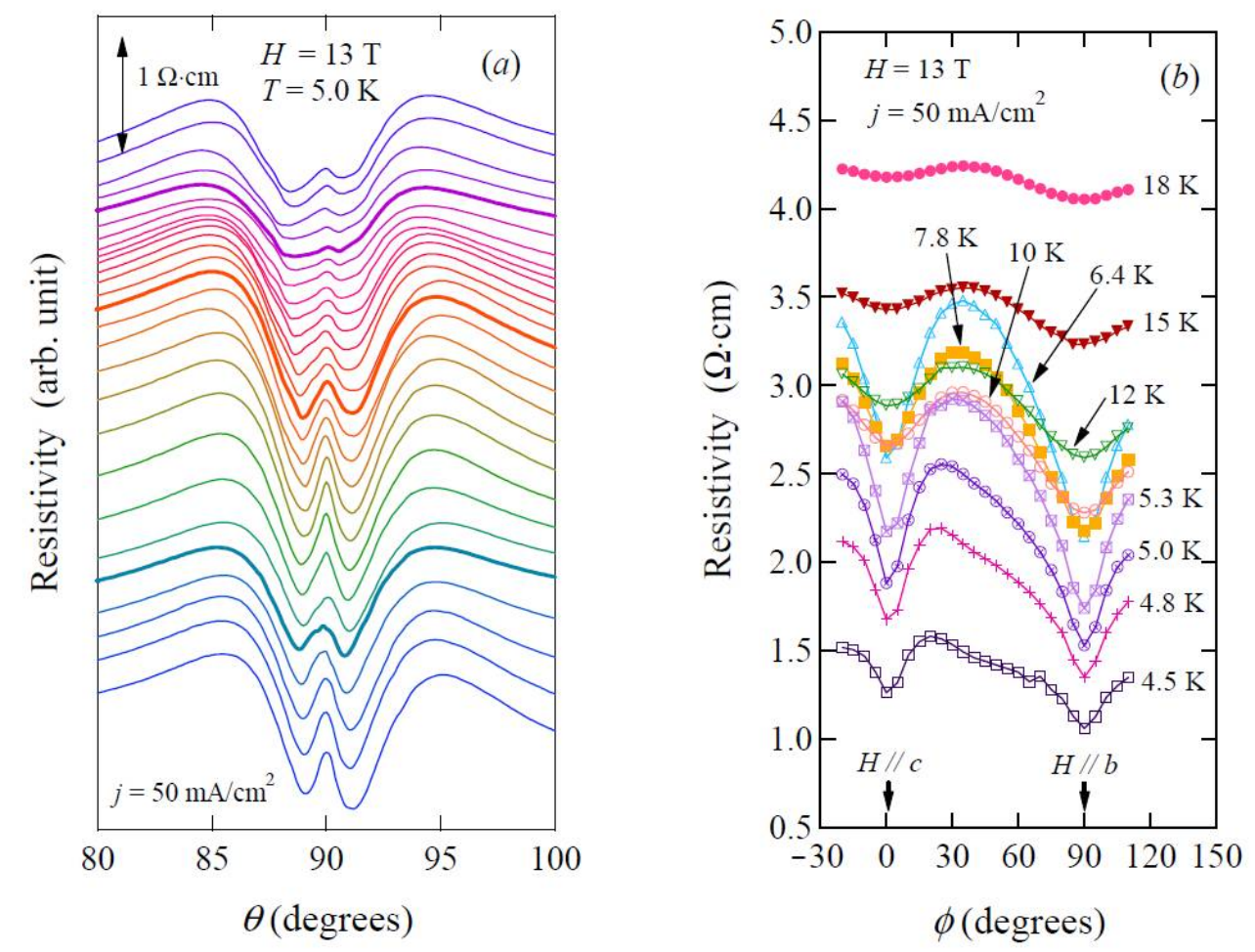

Figure 4. (a) Polar angle dependence of interlayer resistance at $5.0 \mathrm{~K}$ under a magnetic field of $13 \mathrm{~T}$ for various values of $\phi$. The curves are measured in intervals of $\Delta \phi=5^{\circ}$ between $120^{\circ}$ (top curve) and $-20^{\circ}$ (bottom). (b) Dependence of resistivity at $\theta=90^{\circ}$ at various temperatures. (Reprinted from [25]. Copyright 2013 The Physical Society of Japan.)

As seen in Figure $4 b$, we observe sharp peaks related to dissipative feature arising from the vortex dynamics at any azimuth direction within the conducting plane. Therefore, the origin of the cusp-like minima is discussed in terms of the anisotropic vortex dynamics. Since the Josephson vortex essentially does not have a normal core, the pinning effect arising from lattice defects and/or impurities is negligibly small for vortex motion parallel to the conducting layers. In this situation, the friction force is largely affected by the damping viscosity, which depends on the energy dissipation processes of QPs. In the simple description of flux-flow behavior, the Lorentz force on a vortex is balanced by a viscous drag force. Since the Lorentz force keeps constant during the field direction changed, the anisotropic flux-flow resistivity observed here suggests a remarkable variation of the QP excitations by the applied field rotating within the conducting layers because flux-flow resistance can be regarded as a measure of QP dissipation in the vortex dynamics [20-24].

Let us discuss the mechanism of the observed fourfold oscillation in terms of the Doppler effect originally proposed by Volovik $[17,18]$. In the $d$-wave paring state, a nonzero QP density-of-state (DOS) is generated on the Fermi surface by the effect of Doppler shifted state. Theoretical studies [18] clearly show that the DOS possesses a broad maximum for the field parallel to the antinodal direction and sharp minimum for the field along the node. When an applied field is parallel to the antinodal orientation, all four nodes affect the DOS. On the other hand, when the field is parallel to a nodal orientation, QPs at that node do not affect the DOS. Since a damping of the Josephson vortex motion by QPs is expected, the stronger damping will occur when the magnetic field is applied parallel to the antinodal orientations, leading to the suppression of the flux-flow resistivity for the antinodal orientation. When the $d_{x}{ }^{2}-y^{2}$ pairing is assumed, $\rho(\phi)$ should become minima at $\phi=0^{\circ}(\mathrm{H} / / \mathrm{c})$ and $\phi=90^{\circ}(\mathrm{H} / / \mathrm{b})$ and maximum at $\phi=45^{\circ}$, which agrees with Figure $4 \mathrm{~b}$. Thus, we consider that our result concerned with flux-flow resistance is consistent with $d_{x}^{2}-y^{2}$ pairing state, as suggested by STM [13] and thermal conductivity experiments [14].

The above consideration is essentially based on the semiclassical theory $[17,18]$. Unfortunately, this semiclassical treatment is valid only at low temperatures and low fields and 
does not consider the scattering effect between the QPs and moving vortices. For wider temperature and field ranges, Vorontsov and Vekhter [46,47] developed a microscopic theory for the angular dependent properties of thermal conductivity and heat capacity in $d$-wave SC state. They found that application of the magnetic field parallel to the SC gap nodes may result in maxima or minima in their angular dependence, depending upon the location in the $T-H$ plane. This variation is attributable to the scattering of the QPs on vortices. Such sign reversal oscillation was observed and discussed in the heavy fermion

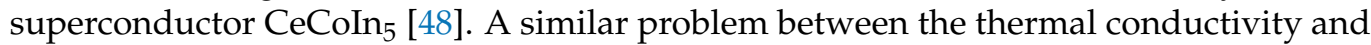
heat capacity measurements was discussed for $\mathrm{K}-(\mathrm{ET})_{2} \mathrm{Cu}(\mathrm{NCS})_{2}[14,15]$. According to the $T-H$ diagrams in $[46,47]$, field-angle dependences of heat capacity and thermal conductivity do not show sign reversal pattern at $H / H_{\mathrm{c} 2} \sim 0.43$ and $T / T_{\mathrm{c}}>0.48$, where our experimental study was done. Although our results are not inconsistent with no sign reversal oscillation for a wide temperature region, further experimental study such as confirmation of sign reversal pattern at lower fields will be needed to elucidate the effect of the Doppler shifted state on the vortex dynamics.

\section{2. $\beta^{\prime \prime}-(E T)_{2} \mathrm{SF}_{5} \mathrm{CH}_{2} \mathrm{CF}_{2} \mathrm{SO}_{3}$}

The crystal structure of $\beta^{\prime \prime}-(\mathrm{ET})_{2} \mathrm{SF}_{5} \mathrm{CH}_{2} \mathrm{CF}_{2} \mathrm{SO}_{3}\left(T_{\mathrm{C}}=5.2 \mathrm{~K}\right)$ has triclinic symmetry and the ET donor molecules form 2D conduction layers parallel to the $a-b$ plane [49]. The insulating $\mathrm{SF}_{5} \mathrm{CH}_{2} \mathrm{CF}_{2} \mathrm{SO}_{3}{ }^{-}$layer is intercalated between the ET donor layers, which makes the $c^{*}$-axis the least conducting direction. Both a 2D Fermi pocket and a pair of quasi-1D Fermi sheets is predicted from the band-structure calculation [50]. The SdH effect and AMRO (angular-dependent magnetoresistance oscillation) studies clearly show one small 2D FS with an area of $5 \%$ of the first Brillouin zone [50,51]. Reflecting the layered structure, its GL coherence length perpendicular to the conducting layers, $\xi_{\perp}(0)(\sim 7.9 \pm 1.5 \AA)$, is shorter than the interlayer spacing $d$ of $17.5 \AA$ [28].

There are three intriguing properties in $\beta^{\prime \prime}-(\mathrm{ET})_{2} \mathrm{SF}_{5} \mathrm{CH}_{2} \mathrm{CF}_{2} \mathrm{SO}_{3}$. First, high field $\mathrm{AMRO}$ showed the nature of incoherent interlayer transport. This means that the FS of $\beta^{\prime \prime}-(\mathrm{ET})_{2} \mathrm{SF}_{5} \mathrm{CH}_{2} \mathrm{CF}_{2} \mathrm{SO}_{3}$ is regarded as the highly $2 \mathrm{D}$ confined electron system [52]. Second, the FS structure is very similar to that of $\mathrm{K}-(\mathrm{ET})_{2} \mathrm{Cu}(\mathrm{NCS})_{2}$ [49]. Third and most importantly, the SC state of $\beta^{\prime \prime}-(\mathrm{ET})_{2} \mathrm{SF}_{5} \mathrm{CH}_{2} \mathrm{CF}_{2} \mathrm{SO}_{3}$ may be mediated by charge fluctuations because a pressure-induced charge ordering state is observed at around 1 GPa [53]. For $\beta$ " type organic conductors, a large intersite Coulomb repulsion has been theoretically predicted $[6,8,54]$. Moreover, $d$-wave superconductivity mediated by charge fluctuations has been proposed based on above theoretical study [8,55], but not confirmed experimentally. In this section, we discuss the relationship between in-plane angular variation of $H_{\mathrm{c} 2}$ and vortex dynamics in $\beta^{\prime \prime}-(\mathrm{ET})_{2} \mathrm{SF}_{5} \mathrm{CH}_{2} \mathrm{CF}_{2} \mathrm{SO}_{3}$.

\subsubsection{Anisotropy of Upper Critical Field in $\beta^{\prime \prime}-(\mathrm{ET})_{2} \mathrm{SF}_{5} \mathrm{CH}_{2} \mathrm{CF}_{2} \mathrm{SO}_{3}$}

To study the upper critical field, $H_{\mathrm{c} 2}(\phi)$, within the conducting $a-b$ plane, the interlayer resistance as a function of magnetic field at various fixed $\phi$ was investigated as shown by Figure 5a. The resistance curves were taken in intervals of $\Delta \phi=10^{\circ}$. At around the SC transition, the resistance gradually reaches zero with decreasing magnetic field. Figure $5 \mathrm{~b}$ presents the in-plane angular variation of $H_{\mathrm{c} 2}$. We define $H_{\mathrm{c} 2}$, when $R / R_{n}=0.9,0.7$, and 0.5 , where $R_{n}$ is the normal state resistance given by extrapolating $R(H)$ in the higher field region of $13 \leqq \mu_{0} H \leqq 14 \mathrm{~T}$. It is clear that the angular variation of $H_{\mathrm{c} 2}$ exhibits maxima at around $\phi= \pm 90^{\circ}$ and $\phi=0^{\circ}$. Although the values of $H_{\mathrm{c} 2}$ are changed by the different criteria, the fourfold oscillation pattern of $H_{\mathrm{c} 2}$ itself remains. 

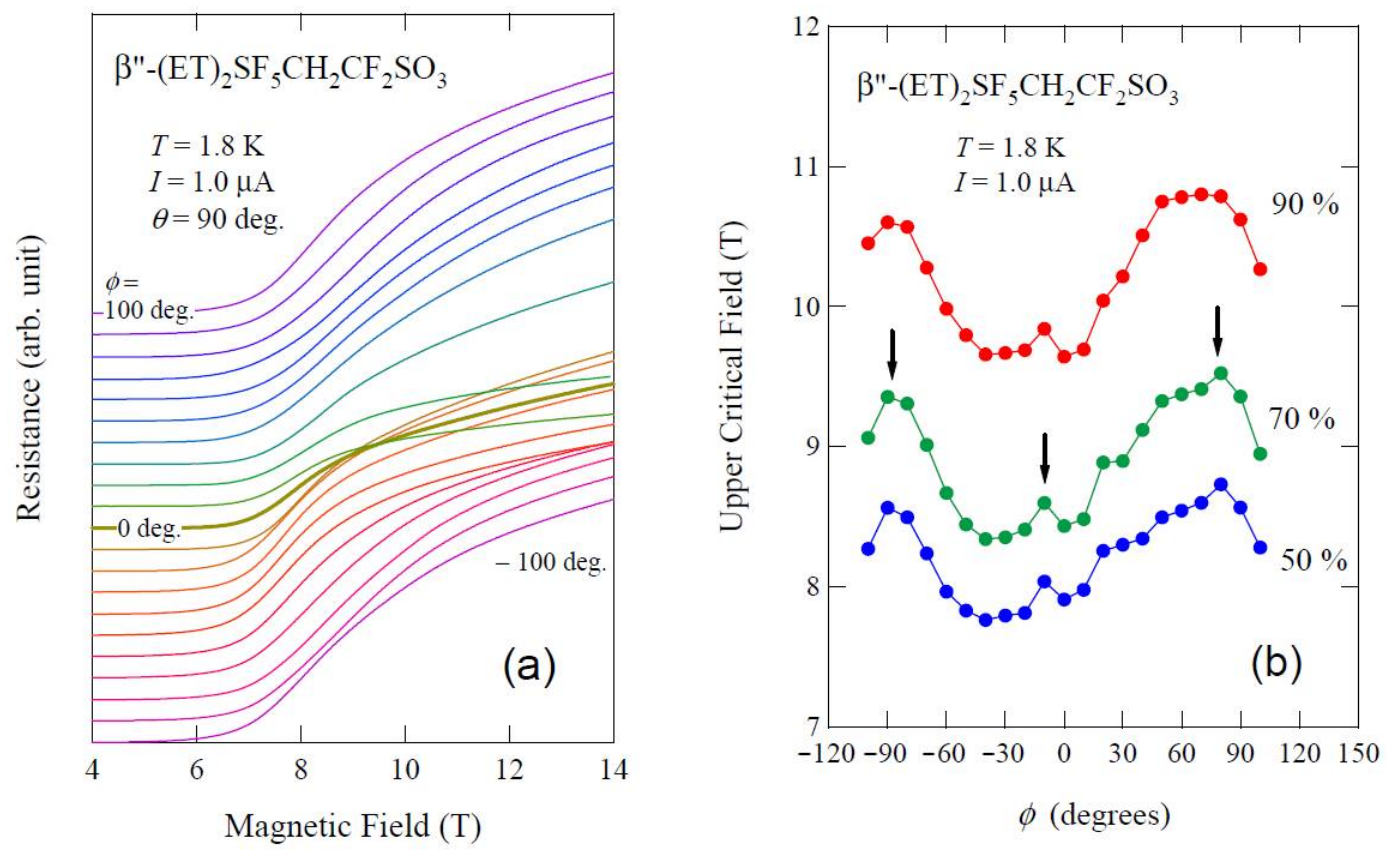

Figure 5. (a) Magnetic field dependence of interlayer resistance at various fixed $\phi$ in the ohmic regime, where applied field is parallel to the conducting plane. The curves are taken from $\phi=100^{\circ}$ (top curve) to $-100^{\circ}$ (bottom) with intervals of $\Delta \phi=10^{\circ}$. (b) In-plane angular variation of $H_{\mathrm{c} 2}$ determined from the resistive transition. The $H_{\mathrm{c} 2}$ values are defined as the fields at which the resistance of the measured sample has reached $90 \%, 70 \%$, and $50 \%$ of its normal-state value. (Reprinted from [27]. Copyright 2015 The Physical Society of Japan.)

For an unconventional superconductor, $H_{\mathrm{c} 2}(\phi)$ minima occur for applied field parallel to the nodal directions. Thus, it is considered that the SC gap possesses its node (or minimum) at approximately $\pi / 4$ from the $b$-axis. This result is in favor of a $d_{x}^{2}-y^{2}$ gap symmetry [38].

\subsubsection{In-Plane Anisotropy of Vortex Dynamics in $\beta^{\prime \prime}-(\mathrm{ET})_{2} \mathrm{SF}_{5} \mathrm{CH}_{2} \mathrm{CF}_{2} \mathrm{SO}_{3}$}

To further discuss observed $d$-wave like anisotropy of $H_{\mathrm{c} 2}$, the in-plane anisotropy of the vortex dynamics is next shown. Figure 6 presents the polar angle dependence of the interlayer resistance at various $\phi$ values. The structures around $\theta=90^{\circ}$ (i.e., dip or peak) depend on $\phi$, showing the anisotropic vortex dynamics within the conducting layers. To see the anisotropic field effect, the $\phi$-dependence of the interlayer resistance at $\theta=90^{\circ}$ for various currents is shown in Figure 7a. At $100 \mu \mathrm{A}$, we observe a fourfold angular oscillation: cusp-like minima are observed at $\phi=20^{\circ}$ and $\pm 90^{\circ}$. With increasing current, the amplitude increases, showing the remarkable non-ohmic transport phenomena. Figure $7 \mathrm{~b}$ presents $\phi$-dependence of the interlayer resistance at $\theta=90^{\circ}$ for several magnetic fields. As shown by Figure $7 \mathrm{~b}$, the effect of magnetic field on the flux-flow resistance is very similar to Figure 7a. With increasing magnetic field, a non-sinusoidal fourfold angular pattern is found. The cusp-like minima are observed at $\phi=20^{\circ}$ and $\pm 90^{\circ}$ that are the same as Figure 7a. The current dependence of the flux-flow resistance at a fixed field strength (Figure 7a) is very similar to the field dependence of that at a fixed current (Figure 7b), suggesting that combination of high magnetic field and large current, that is, Lorentz force plays an important role for appearance of the fourfold pattern in the flux-flow resistance. Similar fourfold pattern has been found in the flux-flow resistance in K-(ET) ${ }_{2} \mathrm{Cu}(\mathrm{NCS})_{2}$ [25]. 


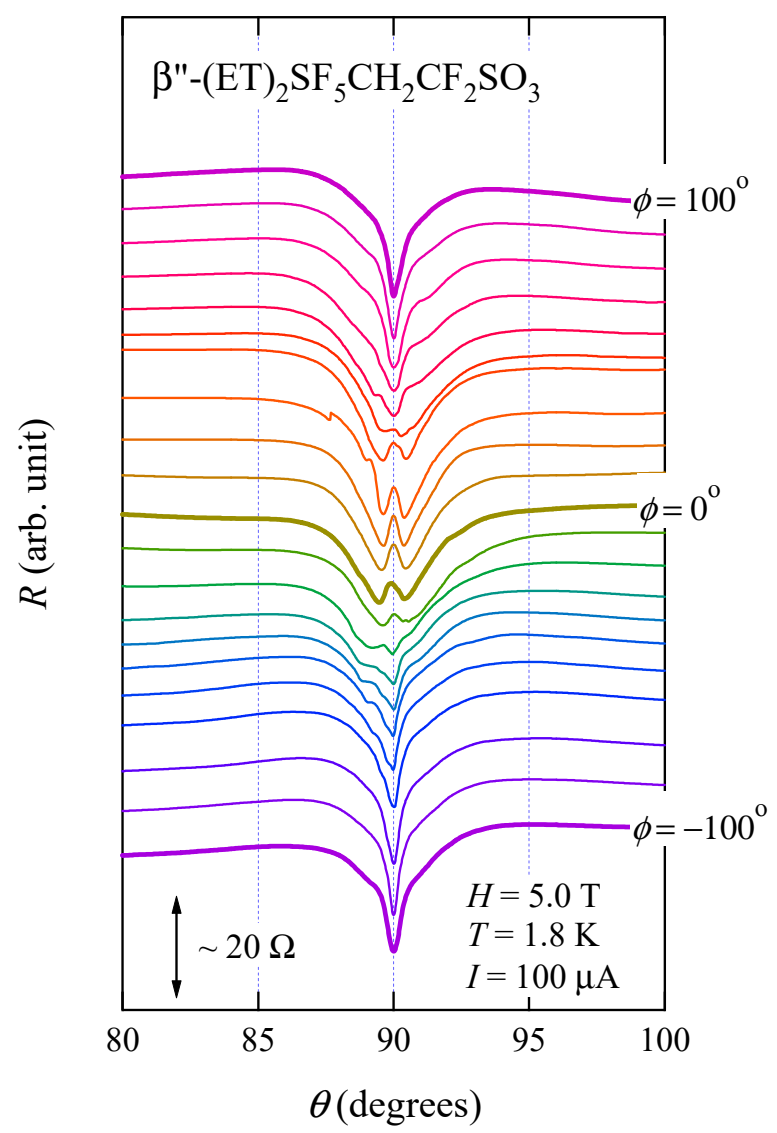

Figure 6. Polar angle dependence of interlayer resistance for various fixed $\phi$ values. The curves are taken in intervals of $\Delta \phi=10^{\circ}$ between $\phi=100^{\circ}$ (top curve) and $-100^{\circ}$ (bottom). (Reprinted from [27]. Copyright 2015 The Physical Society of Japan.).
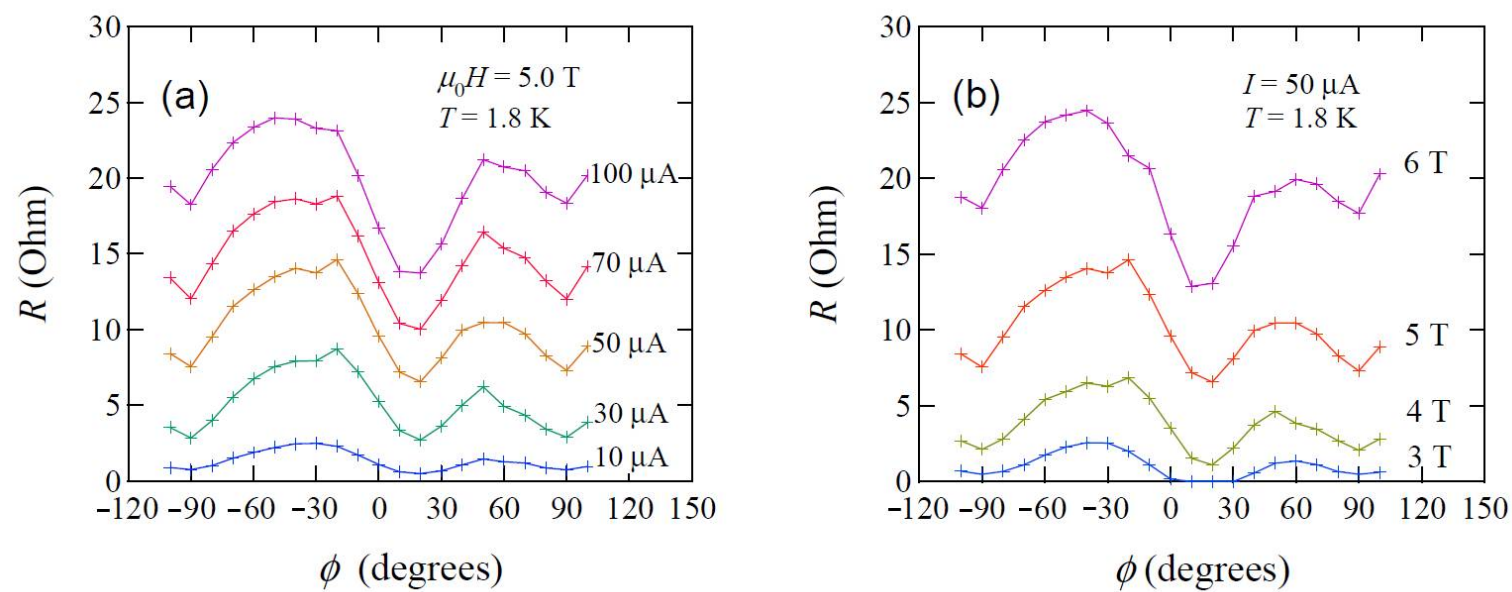

Figure 7. Azimuth angle dependence of the flux-flow resistance for several values of current (a) and of magnetic field (b). (Reprinted from [27]. Copyright 2015 The Physical Society of Japan.)

According to theoretical studies [18,46,47], the Doppler effect in the $d$-wave paring state gives rise to remarkable response of QP generation on the Fermi surface at low temperatures. It is known that as a field is parallel to the antinodal orientation, QPs are excited at four nodes that contribute to the DOS. On the other hand, when the field is directed along a nodal orientation, QPs at that node do not contribute to the DOS, and therefore the Doppler shift vanishes at these points. Since the QPs are expected to dampen the vortex motion, the flux-flow resistance associated with the vortex motion will 
be suppressed for the magnetic field applied parallel to the antinodal directions, giving rise to the weaker FFR for the antinodal orientation. For $\beta^{\prime \prime}-(\mathrm{ET})_{2} \mathrm{SF}_{5} \mathrm{CH}_{2} \mathrm{CF}_{2} \mathrm{SO}_{3}$, when $d_{x}{ }^{2}-y^{2}$ pairing, as discussed in Figure $4 \mathrm{~b}$, is assumed, $\mathrm{R}(\phi)$ should have minima at around $\phi= \pm 90^{\circ}$ and $\phi=0^{\circ}$ and it should have maxima at around $\phi= \pm 45^{\circ}$ orientations, and therefore this is consistent with Figure 7a,b.

Finally, a comment is given on the paring mechanism for the SC state in $\beta^{\prime \prime}$ (ET) ${ }_{2} \mathrm{SF}_{5} \mathrm{CH}_{2} \mathrm{CF}_{2} \mathrm{SO}_{3}$. The nodal orientations discussed here are far from the orientations of most effective nesting vector on the Fermi surface predicted from the band-structure calculation, as well as determined from the AMRO experiments [26,50,51]. If a spin fluctuation scenario is assumed, it is natural to expect the nodes that are parallel to the antiferromagnetic nesting vector, to be along the $b^{*}$ and $a^{*}$ (or $a$ ) orientations. It is intriguing to point out that superconductivity with another $d$-wave symmetry is theoretically suggested based on the charge fluctuation scenario [55]. Further investigation is needed to elucidate which mechanism (i.e., spin fluctuations versus charge fluctuations) is more likely.

\section{3. $\lambda$-(BETS $)_{2} \mathrm{GaCl}_{4}$}

The layered organic conductor $\lambda$-(BETS) ${ }_{2} \mathrm{GaCl}_{4}$ undergoes a SC transition at $T_{\mathrm{c}}$ of $\sim 8 \mathrm{~K}$ [56]. The BETS donor molecules are stacked along the $a$ - and $c$-axes. The insulating $\mathrm{GaCl}_{4}{ }^{-}$anion layers are inserted between the BETS conducting layers. Thus, the $2 \mathrm{D}$ conducting layers are formed in $\lambda$-(BETS $)_{2} \mathrm{GaCl}_{4}$. Reflecting the layered structure, its GL coherence length perpendicular to the layers is shorter than the interlayer spacing of $18.6 \AA$ [57]. $\lambda$-(BETS $)_{2} \mathrm{GaCl}_{4}$ is known as a good candidate for realizing the FuldeFerrell-Larkin-Ovchinnikov state [57,58]. Another intriguing point is that its isostructural compound $\lambda$-(BETS $)_{2} \mathrm{FeCl}_{4}$ shows a field-induced SC transition [59]. Band-structure calculation [56] predicts the existence of one closed 2D Fermi pocket and two 1D Fermi sheets that are topologically the same as K-(ET) ${ }_{2} \mathrm{Cu}(\mathrm{NCS})_{2}[50,51]$. Measurements of the $\mathrm{SdH}$ and AMROs are qualitatively consistent with the band calculation [60].

As for the SC gap structure, a STM experiment suggested $d_{x y}$-wave symmetry with the line nodes along the $a^{*}$ - and $c^{*}$-axes [61]. From systematic investigations by chemical substitution in the anions [62,63] or by selecting different donor molecules [64], the SC phase is suggested to exist next to the Mott insulating phase, which is similar to the K$(\mathrm{ET})_{2} \mathrm{X}$ system [65]. An NMR study showed the development of spin fluctuations beyond the SC phase transition temperature [66]. A heat capacity study showed a $d$-wave pairing state [67], whereas a $\mu \mathrm{SR}$ study clamed a mixture of the extended $s$ - and $d$-wave SC gap [68]. In this section, we discuss the interplay between in-plane anisotropy of vortex dynamics and the SC gap structure for $\lambda$-(BETS) ${ }_{2} \mathrm{GaCl}_{4}[26]$.

\section{In-Plane Anisotropy of Vortex Dynamics in $\lambda$-(BETS) ${ }_{2} \mathrm{GaCl}_{4}$}

Figure 8 shows the polar angle dependence of the interlayer resistance at various fixed $\phi$ values. The sharp peak is clearly observed at $\theta=90^{\circ}$ at all $\phi$ values, which shows the vortex dynamics for all $\phi$-directions. Figure 9 shows the azimuth angle dependence of the interlayer resistance at $\theta=90^{\circ}$ for various currents. The in-plane angular dependence is mainly represented by the twofold symmetry with the sharp dip at $\phi=0^{\circ}(\mathrm{H} / / \mathrm{c})$. This feature differs from the cases of K-(ET) ${ }_{2} \mathrm{Cu}(\mathrm{NCS})_{2}$ [25] and $\beta$ "-(ET) ${ }_{2} \mathrm{SF}_{5} \mathrm{CH}_{2} \mathrm{CF}_{2} \mathrm{SO}_{3}$ [27] even though the FS geometry of $\lambda$-(BETS) ${ }_{2} \mathrm{GaCl}_{4}$ [56] is similar to that of $\mathrm{K}-(\mathrm{ET})_{2} \mathrm{Cu}(\mathrm{NCS})_{2}$ [60] and $\beta^{\prime \prime}-(\mathrm{ET})_{2} \mathrm{SF}_{5} \mathrm{CH}_{2} \mathrm{CF}_{2} \mathrm{SO}_{3}[50,51]$. 


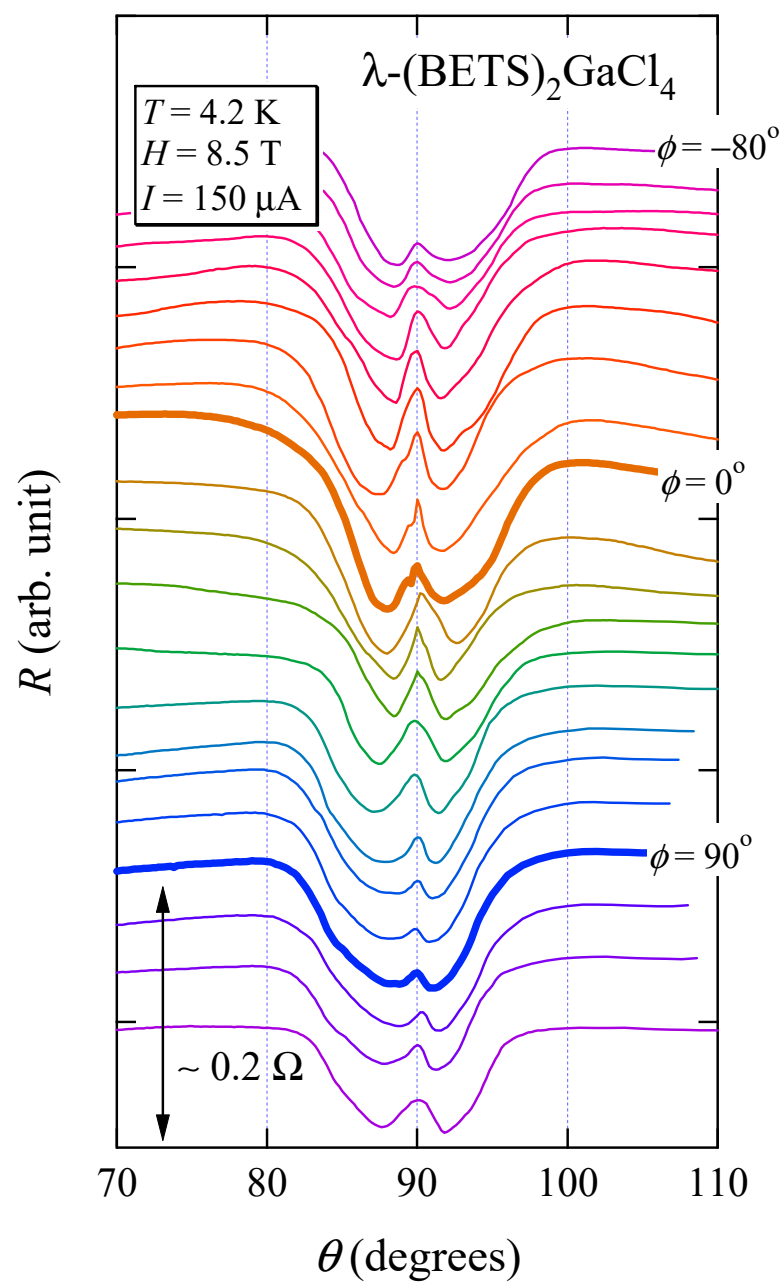

Figure 8. Polar angle dependence of interlayer resistance under rotating field of $8.5 \mathrm{~T}$ for various fixed $\phi$. The curves are taken in intervals of $\Delta \phi=10^{\circ}$ between $-80^{\circ}$ (top curve) and $120^{\circ}$ (bottom). (Reprinted from [26]. Copyright 2014 The Physical Society of Japan.)

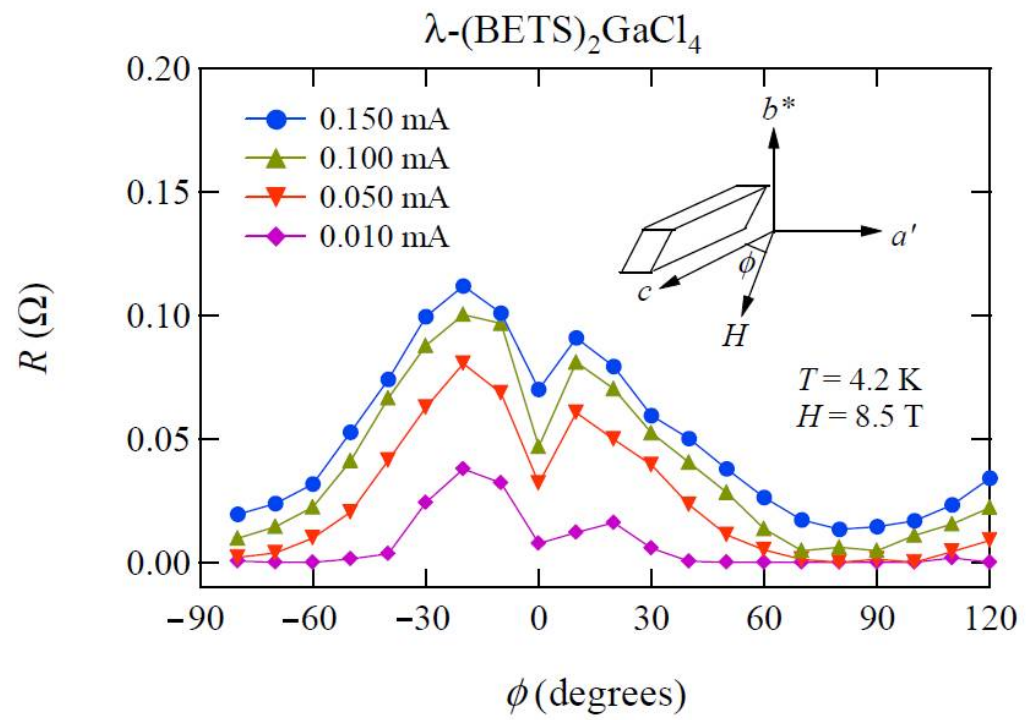

Figure 9. Azimuth angle dependence of flux-flow resistance in $\lambda-(\mathrm{BETS})_{2} \mathrm{GaCl}_{4}$ at various currents. A sharp minimum is observed at $\phi=0^{\circ}(H / / c)$. (Reprinted from [26]. Copyright 2014 The Physical Society of Japan.) 
In spite of the similar FS geometry, we found an in-plane twofold flux-flow resistance anisotropy in $\lambda$-(BETS $)_{2} \mathrm{GaCl}_{4}$ while fourfold-symmetric flux-flow resistance for $\beta^{\prime \prime}$ (ET) ${ }_{2} \mathrm{SF}_{5} \mathrm{CH}_{2} \mathrm{CF}_{2} \mathrm{SO}_{3}$ and K-(ET) $)_{2} \mathrm{Cu}(\mathrm{NCS})_{2}$. As a possible explanation, we consider that the different anisotropic feature may be related to the difference of the interlayer coupling strength [31]. The anisotropy parameter $\Gamma$, given by $\left(\xi_{/} / / \xi_{\perp}\right)^{2}$, for $\beta^{\prime \prime}-(\mathrm{ET})_{2} \mathrm{SF}_{5} \mathrm{CH}_{2} \mathrm{CF}_{2} \mathrm{SO}_{3}$ is $\Gamma \sim 330$ that is larger the those of $\mathrm{K}-(\mathrm{ET})_{2} \mathrm{Cu}(\mathrm{NCS})_{2}(\Gamma \sim 100)$ and $\lambda-(\mathrm{BETS})_{2} \mathrm{GaCl}_{4}$ $(\Gamma \sim 60)$ [27]. Since $\lambda$-(BETS $)_{2} \mathrm{GaCl}_{4}$ is more three dimensional than $\mathrm{K}-(\mathrm{ET})_{2} \mathrm{Cu}(\mathrm{NCS})_{2}$ and $\beta^{\prime \prime}$-(ET) ${ }_{2} \mathrm{SF}_{5} \mathrm{CH}_{2} \mathrm{CF}_{2} \mathrm{SO}_{3}$, an orbital pair breaking effect in $\lambda-(\mathrm{BETS})_{2} \mathrm{GaCl}_{4}$ is stronger than in $\mathrm{K}-(\mathrm{ET})_{2} \mathrm{Cu}(\mathrm{NCS})_{2}$ and $\beta^{\prime \prime}-(\mathrm{ET})_{2} \mathrm{SF}_{5} \mathrm{CH}_{2} \mathrm{CF}_{2} \mathrm{SO}_{3}$.

Due to the orbital pair breaking effect in $\lambda$-(BETS $)_{2} \mathrm{GaCl}_{4}$, the superposition of the normal-state magnetoresistance cannot be avoided in the in-plane anisotropy of the fluxflow resistance. Figure 10 presents the in-plane angular dependence of normal-state magnetoresistance in the magnetic field of $14.8 \mathrm{~T}$ [26]. The normal-state magnetoresistance possesses the twofold symmetry with broad maximum at around $\phi=-20^{\circ}$. The result is consistent with Tanatar et al. [57]. The twofold symmetric normal-state magnetoresistance can be understood in terms of Fermi-surface anisotropy if it mainly originates from the ellipsoidal 2D pocket elongated along the $c$-axis. In the small $\Gamma$ system, the large twofold component in the normal-state magnetoresistance may mask the fourfold ones.

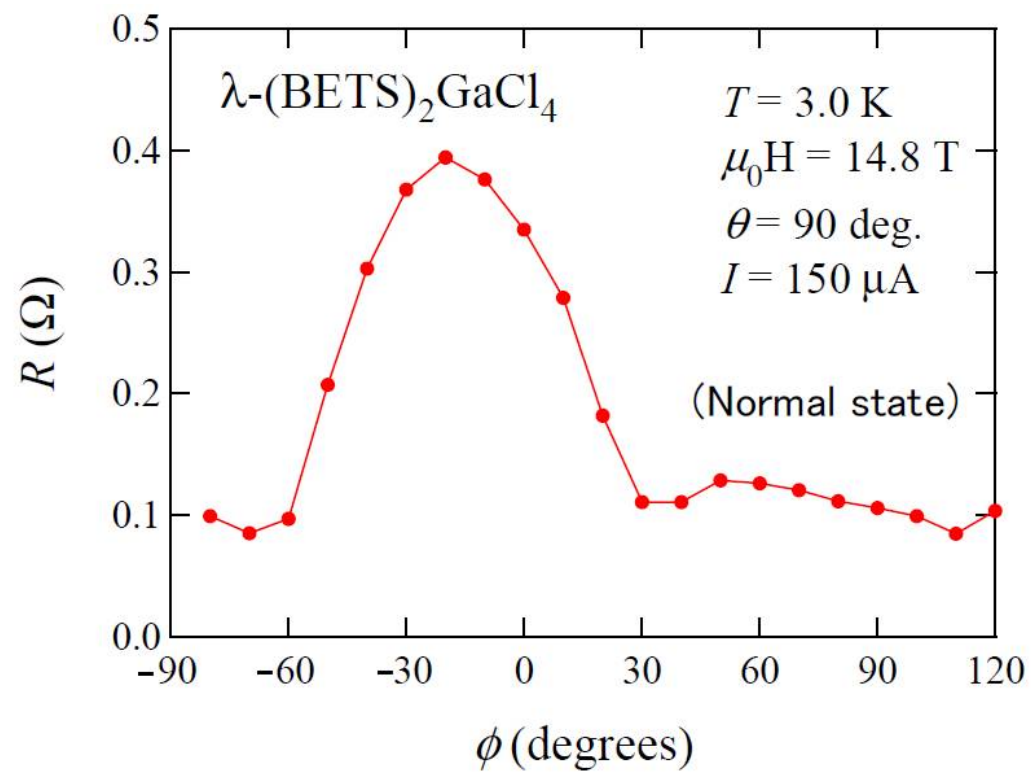

Figure 10. Azimuth angle dependence of normal-state magnetoresistance in $\lambda-(\mathrm{BETS})_{2} \mathrm{GaCl}_{4}$ within the conducting plane. (Reprinted from [26]. Copyright 2014 The Physical Society of Japan.)

The origins of the sharp dip $\left(\phi=-20^{\circ}\right)$ and the broad minimum (around $\phi=90^{\circ}$ ) in Figure 9 are next discussed. Similar dip structures are found in $\mathrm{K}-(\mathrm{ET})_{2} \mathrm{Cu}(\mathrm{NCS})_{2}$ and $\beta^{\prime \prime}$-(ET) $)_{2} \mathrm{SF}_{5} \mathrm{CH}_{2} \mathrm{CF}_{2} \mathrm{SO}_{3}$. The origin of the dip in $\lambda$-(BETS) ${ }_{2} \mathrm{GaCl}_{4}$ should be the same as that in $\mathrm{K}-(\mathrm{ET})_{2} \mathrm{Cu}(\mathrm{NCS})_{2}$ and $\beta^{\prime \prime}-(\mathrm{ET})_{2} \mathrm{SF}_{5} \mathrm{CH}_{2} \mathrm{CF}_{2} \mathrm{SO}_{3}$. To theoretically discuss the $\mathrm{SC}$ gap structure for $\lambda$-(BETS) ${ }_{2} \mathrm{GaCl}_{4}$, Aizawa et al. [69] performed first-principles band calculation. Considering the spin-fluctuation-mediated mechanism, they discussed the SC gap function by applying the random phase approximation. They showed that the obtained SC gap changes its sign four times along the Fermi surface, suggesting a $d$-wave SC gap in $\lambda$ (BETS $)_{2} \mathrm{GaCl}_{4}$. Reflecting the low symmetry of the crystal structure in $\lambda$-(BETS $)_{2} \mathrm{GaCl}_{4}$, however, the SC gap has only twofold symmetry. It means that the predicted SC gap has a large gap between narrow opening nodes with an acute angle (around the steep node structure) and a small gap between wide opening nodes with an obtuse angle. Recent magnetic-field-angle-resolved heat capacity study [70] is consistent with theoretically predicted SC gap function. The large gap is located along the $c$-axis [69] which agrees with the position of sharp dip [26]. The small gap exists at around $a$-axis [69], where 
we observed broad minimum in flux-flow resistance [26]. Thus, experimental results of flux-flow resistance in $\lambda$-(BETS $)_{2} \mathrm{GaCl}_{4}$ [26] are consistent with the $d$-wave gap structure theoretically discussed by Aizawa [69].

\section{Summary}

In order to discuss the relationship between vortex dynamics and the SC gap structure with $d$-wave paring symmetry, we investigated in-plane angular variation of vortex dynamics for the layered organic superconductors K-(ET) ${ }_{2} \mathrm{Cu}(\mathrm{NCS})_{2}, \beta^{\prime \prime}-(\mathrm{ET})_{2} \mathrm{SF}_{5} \mathrm{CH}_{2} \mathrm{CF}_{2} \mathrm{SO}_{3}$, and $\lambda$-(BETS $)_{2} \mathrm{GaCl}_{4}$. We observed clear fourfold-symmetric anisotropy in the interlayer flux-flow resistance for $\mathrm{K}-(\mathrm{ET})_{2} \mathrm{Cu}(\mathrm{NCS})_{2}$ and $\beta^{\prime \prime}-(\mathrm{ET})_{2} \mathrm{SF}_{5} \mathrm{CH}_{2} \mathrm{CF}_{2} \mathrm{SO}_{3}$, while only twofold symmetry in $\lambda$-(BETS $)_{2} \mathrm{GaCl}_{4}$. For K-(ET) $)_{2} \mathrm{Cu}(\mathrm{NCS})_{2}$ and $\beta "$ "-(ET) ${ }_{2} \mathrm{SF}_{5} \mathrm{CH}_{2} \mathrm{CF}_{2} \mathrm{SO}_{3}$, fluxflow resistivity showing fourfold oscillation can be consistently explained by assuming the enhanced viscous motion of vortices by QPs arising from the Doppler effect. Absence of the fourfold anisotropy in $\lambda$-(BETS $)_{2} \mathrm{GaCl}_{4}$ is discussed in the two regimes. The first regime is related to the stronger interlayer coupling in the $\lambda$-(BETS ${ }_{2} \mathrm{GaCl}_{4}$ system as compared with $\mathrm{K}-(\mathrm{ET})_{2} \mathrm{Cu}(\mathrm{NCS})_{2}$ and $\beta^{\prime \prime}-(\mathrm{ET})_{2} \mathrm{SF}_{5} \mathrm{CH}_{2} \mathrm{CF}_{2} \mathrm{SO}_{3}$. The second regime is discussed in terms of recent theoretical study by Aizawa et al. In this scenario, flux-flow resistivity with twofold anisotropy may be associated with the crystal structure with low symmetry, which is rather different from those of $\mathrm{K}-(\mathrm{ET})_{2} \mathrm{Cu}(\mathrm{NCS})_{2}$ and $\beta "-(\mathrm{ET})_{2} \mathrm{SF}_{5} \mathrm{CH}_{2} \mathrm{CF}_{2} \mathrm{SO}_{3}$.

For these three organic superconductors, the origin of the in-plane anisotropy of fluxflow resistance with sharp minima is discussed in terms of the effect of Doppler shifted state. Based on these results, we claim that angular dependence of the vortex dynamics strongly depends on the SC gap structure. We hope that further understanding of the vortex dynamics presented here leads to clarifying the mechanism of the unconventional superconductors in various strongly correlated electron systems.

Funding: These works were partly supported by Grants-in-Aid for Scientific Research on Innovative Areas (Grant No. 20110004) from the Ministry of Education, Culture, Sports, Science, and Technology (MEXT) of Japan, and Grants-in-Aid for Scientific Research (C) (Grant No. 25400383) from the Japan Society for the Promotion of Science (JSPS).

Institutional Review Board Statement: Not applicable.

Informed Consent Statement: Not applicable.

Data Availability Statement: The data presented in this study are available within the article.

Acknowledgments: The author thanks Shinya Uji, Taichi Terashima, Takako Konoike, Satoshi Tsuchiya, Kaori Sugii, Takayuki Isono, Yoritsugu Iida, Kazuya Saito, Yasuhisa Yamamura, Motoi Kimata, Hidetaka Satsukawa, Jun-ichi Yamada, Biao Zhou, Akiko Kobayashi, Hayao Kobayashi, John A. Schlueter, Hirohito Aizawa, Kazuhiko Kuroki, Nobuhiko Hayashi, and Yoichi Higashi for useful discussions and suggestions.

Conflicts of Interest: The author declares no conflict of interest.

\section{References}

1. Ardavan, A.; Brown, S.; Kagoshima, S.; Kanoda, K.; Kuroki, K.; Mori, H.; Ogata, M.; Uji, S.; Wosnitza, J. Recent Topics of Organic Superconductors. J. Phys. Soc. Jpn. 2012, 81, 011012. [CrossRef]

2. Lang, M.; Muller, J. Organic Superconductors. In Superconductivity; Bennemann, K.H., Ketterson, J.B., Eds.; Springer: Berlin/Heidelberg, Germany, 2008; Volume II, pp. 1155-1223.

3. Kanoda, K. Electron correlation, metal-insulator transition and superconductivity in quasi-2D organic systems, (ET) ${ }_{2} \mathrm{X}$. Physica C 1997, 282-287, 299-302. [CrossRef]

4. Sasaki, T.; Yoneyama, N.; Matsuyama, A.; Kobayashi, N. Magnetic and electronic phase diagram and superconductivity in the organic superconductors K-(ET) ${ }_{2}$ X. Phys. Rev. B 2002, 65, 060505. [CrossRef]

5. Clay, R.T.; Li, H.; Mazumdar, S. Absence of Superconductivity in the Half-Filled Band Hubbard Model on the Anisotropic Triangular Lattice. Phys. Rev. Lett. 2008, 101, 166403. [CrossRef] [PubMed]

6. Dayal, S.; Clay, R.T.; Li, H.; Mazumdar, S. Paired electron crystal: Order from frustration in the quarter-filled band. Phys. Rev. B 2011, 83, 245106. [CrossRef] 
7. Dayal, S.; Clay, R.T.; Mazumdar, S. Absence of long-range superconducting correlations in the frustrated half-filled-band Hubbard model. Phys. Rev. B 2012, 85, 165141. [CrossRef]

8. Qin, M.; Chung, C.-M.; Shi, H.; Vitali, E.; Hubig, C.; Schollwöck, U.; White, S.R.; Zhang, S. Absence of Superconductivity in the Pure Two-Dimensional Hubbard Model. Phys. Rev. X 2020, 10, 031016. [CrossRef]

9. Mayaffre, H.; Wzietek, P.; Jérome, D.; Batail, P. Superconducting State of $\mathrm{k}-(\mathrm{ET})_{2} \mathrm{Cu}\left[\mathrm{N}(\mathrm{CN})_{2}\right] \mathrm{Br}$ Studied by ${ }^{13} \mathrm{C}$ NMR: Evidence for Vortex-Core-Induced Nuclear Relaxation and Unconventional Pairing. Phys. Rev. Lett. 1995, 75, 4122-4125. [CrossRef] [PubMed]

10. Nakazawa, Y.; Kanoda, K. Low-temperature specific heat of $\mathrm{k}-(\mathrm{BEDT}-\mathrm{TTF})_{2} \mathrm{Cu}\left[\mathrm{N}(\mathrm{CN})_{2}\right] \mathrm{Br}$ in the superconducting state. Phys. Rev. B 1986, 55, R8670-R8673. [CrossRef]

11. Taylor, O.J.; Carrington, A.; Schlueter, J.A. Specific-Heat Measurements of the Gap Structure of the Organic Superconductors к-(ET) ${ }_{2} \mathrm{Cu}\left[\mathrm{N}(\mathrm{CN})_{2}\right] \mathrm{Br}$ and $\mathrm{k}-(\mathrm{ET})_{2} \mathrm{Cu}(\mathrm{NCS})_{2}$. Phys. Rev. Lett. 2007, 99, 057001. [CrossRef] [PubMed]

12. Schrama, J.M.; Rzepniewski, E.; Edwards, R.S.; Singleton, J.; Ardvan, A.; Kurmoo, M.; Day, P. Millimeter-Wave Magnetooptical Determination of the Anisotropy of the Superconducting Order Parameter in the Molecular Superconductor k-(BEDTTTF $)_{2} \mathrm{Cu}(\mathrm{NCS})_{2}$. Phys. Rev. Lett. 1999, 83, 3041-3044. [CrossRef]

13. Arai, T.; Ichimura, K.; Nomura, K.; Takasaki, S.; Yamada, J.; Nakatsuji, S.; Anzai, H. Tunneling spectroscopy on the organic superconductor к-(BEDT-TTF $)_{2} \mathrm{Cu}(\mathrm{NCS})_{2}$ using STM. Phys. Rev. B 2001, 63, 104518. [CrossRef]

14. Izawa, K.; Yamaguchi, H.; Sasaki, T.; Matsuda, Y. Superconducting Gap Structure of k-(BEDT-TTF) ${ }_{2} \mathrm{Cu}(\mathrm{NCS})_{2}$ Probed by Thermal Conductivity Tensor. Phys. Rev. Lett. 2001, 88, 027002. [CrossRef]

15. Malone, L.; Taylor, O.J.; Schlueter, J.A.; Carrington, A. Location of gap nodes in the organic superconductors $\mathrm{k}-(\mathrm{ET})_{2} \mathrm{Cu}(\mathrm{NCS})_{2}$ and $\mathrm{k}-(\mathrm{ET})_{2} \mathrm{Cu}\left[\mathrm{N}(\mathrm{CN})_{2}\right] \mathrm{Br}$ determined by magnetocalorimetry. Phys. Rev. B 2010, 82, 014522. [CrossRef]

16. Sigrist, M.; Ueda, K. Phenomenological theory of unconventional superconductivity. Rev. Mod. Phys. 1991, 63, 239-311. [CrossRef]

17. Volovik, G.E. Superconductivity with lines of gap nodes: Density of states in the vortex. JETP Lett. 1993, 58, 469-473.

18. Vekhter, I.; Hirschfeld, P.J.; Carbotte, J.P.; Nicol, E.J. Anisotropic thermodynamics of $d$-wave superconductors in the vortex state. Phys. Rev. B 1999, 59, R9023-R9026. [CrossRef]

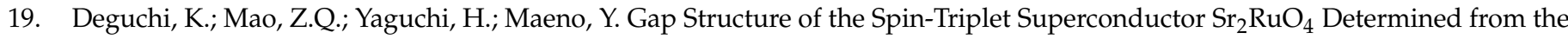
Field-Orientation Dependence of the Specific Heat. Phys. Rev. Lett. 2004, 92, 047002. [CrossRef]

20. Higashi, Y.; Nagai, Y.; Machida, M.; Hayashi, N. Field-angle dependence of the quasiparticle scattering inside a vortex core in unconventional superconductors. Physica C 2011, 471, 828-830. [CrossRef]

21. Higashi, Y.; Nagai, Y.; Machida, M.; Hayashi, N. Effect of uniaxially anisotropic Fermi surface on the quasiparticle scattering inside a vortex core in unconventional superconductors. Physica C 2013, 484, 97-99. [CrossRef]

22. Higashi, Y.; Nagai, Y.; Machida, M.; Hayashi, N. Field-angle resolved flux-flow resistivity as a phase-sensitive probe of unconventional Cooper pairing. Phys. Rev. B 2013, 88, 224511. [CrossRef]

23. Clem, J.R.; Coffey, M.W. Viscous flux motion in a Josephson-coupled layer model of high- $T_{\mathrm{C}}$ superconductors. Phys. Rev. B 1990, 42, 6209-6216. [CrossRef]

24. Koshelev, A.E. Role of in-plane dissipation in dynamics of a Josephson vortex lattice in high-temperature superconductors. Phys. Rev. B 2000, 62, R3616-R3619. [CrossRef]

25. Yasuzuka, S.; Saito, K.; Uji, S.; Kimata, M.; Satsukawa, H.; Terashima, T.; Yamada, J.-I. Anisotropic Josephson-Vortex Dynamics in Layered Organic Superconductor with $d$-Wave Pairing Symmetry. J. Phys. Soc. Jpn. 2013, 82, 064716. [CrossRef]

26. Yasuzuka, S.; Uji, S.; Terashima, T.; Tsuchiya, S.; Sugii, K.; Zhou, B.; Kobayashi, A.; Kobayashi, H. In-Plane Anisotropy of Flux-Flow Resistivity in Layered Organic Superconductor $\lambda$-(BETS) ${ }_{2} \mathrm{GaCl}_{4}$. J. Phys. Soc. Jpn. 2014, 83, 013705. [CrossRef]

27. Yasuzuka, S.; Uji, S.; Terashima, T.; Sugii, K.; Isono, T.; Iida, Y.; Schlueter, J.A. In-Plane Anisotropy of Upper Critical Field and Flux-Flow Resistivity in Layered Organic Superconductor $\beta^{\prime \prime}-(\mathrm{ET})_{2} \mathrm{SF}_{5} \mathrm{CH}_{2} \mathrm{CF}_{2} \mathrm{SO}_{3}$. J. Phys. Soc. Jpn. 2015, 84, 094709. [CrossRef]

28. Müller, J.; Lang, M.; Steglich, F.; Schlueter, J.A.; Kini, A.M.; Geiser, U.; Mohtasham, J.; Winter, R.W.; Gard, G.L.; Sasaki, T.; et al. Comparative thermal-expansion study of $\beta "-(\mathrm{ET})_{2} \mathrm{SF}_{5} \mathrm{CH}_{2} \mathrm{CF}_{2} \mathrm{SO}_{3}$ and $\mathrm{\kappa}-(\mathrm{ET})_{2} \mathrm{Cu}(\mathrm{NCS})_{2}$ : Uniaxial pressure coefficients of $T_{\mathrm{c}}$ and upper critical fields. Phys. Rev. B 2000, 61, 11739-11744. [CrossRef]

29. Wanka, S.; Hagel, J.; Beckmann, D.; Wosnitza, J.; Schlueter, J.A.; Williams, J.M.; Nixon, P.G.; Winter, R.W.; Garal, G.L. Specific heat and critical field of the organic superconductor $\beta^{\prime \prime}-(\mathrm{ET})_{2} \mathrm{SF}_{5} \mathrm{CH}_{2} \mathrm{CF}_{2} \mathrm{SO}_{3}$. Phys. Rev. B 1997, 57, 3084-3088. [CrossRef]

30. Prozorov, R.; Giannetta, R.W.; Schlueter, J.A.; Kini, A.M.; Mohtasham, J.; Winter, R.W.; Gard, G.L. Unusual temperature dependence of the London penetration depth in all-organic $\beta^{\prime \prime}-(\mathrm{ET})_{2} \mathrm{SF}_{5} \mathrm{CH}_{2} \mathrm{SF}_{2} \mathrm{SO}_{3}$ single crystals. Phys. Rev. B 2001, $63,052506$. [CrossRef]

31. Mielke, C.; Singleton, J.; Nam, M.-S.; Harrison, N.; Agosta, C.C.; Fravel, B.; Montgomery, L.K. Superconducting properties and Fermi-surface topology of the quasi-two-dimensional organic superconductor $\lambda$-(BETS) ${ }_{2} \mathrm{GaCl}_{4}$. J. Phys. Condens. Matter 2001, 13, 8325-8345. [CrossRef]

32. Sari, D.P.; Naito, R.; Hiraki, K.; Nakano, T.; Hagiwara, M.; Nozue, Y.; Kusakawa, T.; Hori, A.; Watanabe, I.; Ishii, Y. Anisotropy of Lower Critical Field in Organic Layered Superconductor $\lambda$-(BETS) ${ }_{2} \mathrm{GaCl}_{4}$. Key Eng. Mater. 2020, 860, 137-141. [CrossRef]

33. Saito, G.; Yamochi, H.; Nakamura, T.; Komatsu, T.; Ishiguro, T.; Nogami, Y.; Ito, Y.; Mori, H.; Oshima, K.; Nakashima, M.; et al. Overview of organic superconductor $\mathrm{k}-(\mathrm{BEDT}-\mathrm{TTF})_{2}\left[\mathrm{Cu}(\mathrm{NCS})_{2}\right]$ and its related materials. Synth. Met. 1991, 42, 1993-1998. [CrossRef]

34. Chaparala, M.; Chung, O.H.; Ren, Z.F.; White, M.; Coppens, P.; Wang, J.H.; Hope, A.P.; Naughton, M.J. Vortex-state resistance near parallel orientation in layered superconductors. Phys. Rev. B 1996, 53, 5818. [CrossRef] [PubMed] 
35. Uji, S.; Terashima, T.; Nishimura, M.; Konoike, T.; Enomoto, K.; Cui, H.; Kobayashi, H.; Kobayashi, A.; Tanaka, H.; Tokumoto, M.; et al. Vortex Dynamics and the Fulde-Ferrell-Larkin-Ovchinnikov State in a Magnetic-Field-Induced Organic Superconductor. Phys. Rev. Lett. 2006, 97, 157001. [CrossRef]

36. Yasuzuka, S.; Uji, S.; Satsukawa, H.; Kimata, M.; Terashima, T.; Koga, H.; Yamamura, Y.; Saito, K.; Akutsu, H.; Yamada, J. Anisotropic Josephson-vortex dynamics in layered organic superconductors. Physica B 2010, 405, S288-S290. [CrossRef]

37. Feinberg, D.; Villard, C. Intrinsic pinning and lock-in transition of flux lines in layered type-II superconductors. Phys. Rev. Lett. 1990, 65, 919-922. [CrossRef] [PubMed]

38. Mansky, P.A.; Chaikin, P.M.; Haddon, R.C. Vortex lock-in state in a layered superconductor. Phys. Rev. Lett. 1993, 70, 1323-1326. [CrossRef]

39. Nam, M.-S.; Symington, J.A.; Singleton, J.; Blundell, S.; Ardavan, A.; Perenboom, J.A.A.J.; Kurmoo, M.; Day, P. Angle dependence of the upper critical field in the layered organic superconductor k-(BEDT-TTF) ${ }_{2} \mathrm{Cu}(\mathrm{NCS})_{2}$ (BEDT-TTF $\equiv$ bis(ethylenedithio)tetrathiafulvalene). J. Phys. Condens. Matter. 1999, 11, L477-L484. [CrossRef]

40. Klemm, R.A.; Luther, A.; Beasley, M.R. Theory of the upper critical field in layered superconductors. Phys. Rev. B 1975, 12, 877-891. [CrossRef]

41. Schneider, T.; Schmidt, A. Dimensional crossover in the upper critical field of layered superconductors. Phys. Rev. B 1993, 47, 5915-5921. [CrossRef]

42. Oshima, K.; Urayama, H.; Yamochi, H.; Saito, G. Peculiar Critical Field Behaviour in the Recectly Discovered Ambient Pressure Organic Superconductor (BEDT-TTF $)_{2} \mathrm{Cu}(\mathrm{NCS})_{2}\left(T_{\mathrm{c}}=10.4 \mathrm{~K}\right)$. J. Phys. Soc. Jpn. 1988, 57, 730-733. [CrossRef]

43. Takanaka, K.; Kuboya, K. Anisotropy of Upper Critical Field and Pairing Symmetry. Phys. Rev. Lett. 1995, 75, 323-325. [CrossRef]

44. Yasuzuka, S.; Koga, H.; Yamamura, Y.; Saito, K.; Uji, S.; Terashima, T.; Akutsu, H.; Yamada, J.-I. Dimensional Crossover and Its Interplay with In-Plane Anisotropy of Upper Critical Field in $\beta$-(BDA-TTP) ${ }_{2} \mathrm{SbF}_{6}$. J. Phys. Soc. Jpn. 2017, 86, 084704. [CrossRef]

45. Yasuzuka, S.; Uji, S.; Sugiura, S.; Terashima, T.; Nogami, Y.; Ichimura, K.; Tanda, S. Highly Isotropic In-plane Upper Critical Field in the Anisotropic s-Wave Superconductor $2 \mathrm{H}-\mathrm{NbSe}_{2}$. J. Supercond. Nov. Magn. 2020, 33, 953-958. [CrossRef]

46. Vorontsov, A.B.; Vekhter, I. Unconventional superconductors under a rotating magnetic field. I. Density of states and specific heat. Phys. Rev. B 2007, 75, 224501. [CrossRef]

47. Vorontsov, A.B.; Vekhter, I. Unconventional superconductors under a rotating magnetic field. II. Thermal transport. Phys. Rev. B 2007, 75, 224502. [CrossRef]

48. An, K.; Sakakibara, T.; Settai, R.; Onuki, Y.; Hiragi, M.; Ichioka, M.; Machida, K. Sign Reversal of Field-Angle Resolved Heat Capacity Oscillations in a Heavy Fermion Superconductor CeCoIn 5 and $d_{x}{ }^{2}-y^{2}$ Pairing Symmetry. Phys. Rev. Lett. 2010, 104, 037002. [CrossRef]

49. Geiser, U.; Schlueter, J.A.; Wang, H.H.; Kini, A.M.; Williams, J.M.; Sche, P.P.; Zakowicz, H.I.; VanZile, M.L.; Dudek, J.D.; Nixon, P.G.; et al. Superconductivity at $5.2 \mathrm{~K}$ in an Electron Donor Radical Salt of Bis(ethylenedithio)tetrathiafulvalene (BEDT-TTF) with the Novel Polyfluorinated Organic Anion $\mathrm{SF}_{5} \mathrm{CH}_{2} \mathrm{CF}_{2} \mathrm{SO}_{3}{ }^{-}$. J. Am. Chem. Soc. 1996, 118, 9996-9997. [CrossRef]

50. Beckmann, D.; Wanka, S.; Wosnitza, J.; Schlueter, J.A.; Williams, J.M.; Nixon, P.G.; Winter, R.W.; Gard, G.L.; Ren, J.; Whangbo, M.-H. Characterization of the Fermi surface of the organic superconductor $\beta$ "- $(\mathrm{ET})_{2} \mathrm{SF}_{5} \mathrm{CH}_{2} \mathrm{CF}_{2} \mathrm{SO}_{3}$ by measurements of Shubnikov-de Haas and angle-dependent magnetoresistance oscillations and by electronic band-structure calculations. Eur. Phys. J. B 1998, 1, 295-300. [CrossRef]

51. Brooks, J.S.; Williams, V.; Choi, E.S.; Graf, D.; Tokumoto, M.; Uji, S.; Zou, F.; Wosnitza, J.; Schlueter, J.A.; Davis, H.; et al. Fermiology and superconductivity at high magnetic fields in a completely organic cation radical salt. New J. Phys. 2006, 8, 255. [CrossRef]

52. Wosnitza, J.; Hagel, J.; Qualls, J.S.; Brooks, J.S.; Balthes, E.; Schweitaer, D.; Schlueter, J.A.; Geiser, U.; Mohtasham, J.; Winter, R.W.; et al. Coherent versus incoherent interlayer transport in layered metals. Phys. Rev. B 2000, 65, R180506. [CrossRef]

53. Hagel, J.; Wosnitza, J.; Pfleiderer, C.; Schlueter, J.A.; Mohtasham, J.; Gard, G.L. Pressure-induced insulating state in an organic superconductor. Phys. Rev. B 2003, 68, 104504. [CrossRef]

54. Calandra, M.; Merino, J.; Mckenzie, R.H. Metal-insulator transition and charge ordering in the extended Hubbard model at one-quarter filling. Phys. Rev. B 2002, 66, 195102. [CrossRef]

55. Merino, J.; Mckenzie, R.H. Superconductivity Mediated by Charge Fluctuations in Layered Molecular Crystals. Phys. Rev. Lett. 2001, 87, 237002. [CrossRef]

56. Kobayashi, H.; Udagawa, T.; Tomita, H.; Bun, K.; Naito, T.; Kobayashi, A. A New Organic Superconductor, $\lambda-(B E D T-T S F){ }_{2}$ GaCl ${ }_{4}$. Chem. Lett. 1993, 22, 1559-1562. [CrossRef]

57. Tanatar, M.A.; Ishiguro, T.; Tanaka, H.; Kobayashi, H. Magnetic field-temperature phase diagram of the quasi-two-dimensional organic superconductor $\lambda$-(BETS) ${ }_{2} \mathrm{GaCl}_{4}$ studied via thermal conductivity. Phys. Rev. B 2002, 66, 134503. [CrossRef]

58. Uji, S.; Kodama, K.; Sugii, K.; Terashima, T.; Yamaguchi, T.; Kurita, N.; Tsuchiya, S.; Konoike, T.; Kimata, M.; Kobayashi, A.; et al. Vortex Dynamics and Diamagnetic Torque Signals in Two Dimensional Organic Superconductor $\lambda$-(BETS $)_{2} \mathrm{GaCl}_{4}$. J. Phys. Soc. Jpn. 2015, 84, 104709. [CrossRef]

59. Uji, S.; Shinagawa, H.; Terashima, T.; Yakabe, T.; Terai, Y.; Tokumoto, M.; Kobayashi, A.; Tanaka, H.; Kobayashi, H. Magnetic-fieldinduced superconductivity in a two-dimensional organic conductor. Nature 2001, 410, 908-910. [CrossRef] 
60. Goddard, P.A.; Blundell, S.J.; Singleton, J.; McDonald, R.D.; Ardavan, A.; Narduzzo, A.; Schlueter, J.A.; Kini, A.M.; Sasaki, T. Angle-dependent magnetoresistance of the layered organic superconductor $\mathrm{K}-\mathrm{ET}_{2} \mathrm{Cu}(\mathrm{NCS})_{2}$ : Simulation and experiment. Phys. Rev. B 2004, 69, 174509. [CrossRef]

61. Clark, K.; Hassanien, A.; Khan, S.; Braun, K.-F.; Tanaka, H.; Hla, S.-W. Superconductivity in just four pairs of (BETS) ${ }_{2} \mathrm{GaCl}_{4}$ molecules. Nat. Nanotechnol. 2010, 5, 261-265. [CrossRef]

62. Kobayashi, H.; Akutsu, H.; Arai, E.; Tanaka, H.; Kobayashi, A. Electric and magnetic properties and phase diagram of a series of organic superconductors $\lambda-\mathrm{BETS}_{2} \mathrm{GaX}_{\mathrm{Z}} \mathrm{Y}_{4-z}$ [BETS = bis(ethylenedithiotetraselenafulvalene; $\mathrm{X}, \mathrm{Y}=\mathrm{F}, \mathrm{Cl}, \mathrm{Br} ; 0<z<2$ )]. Phys. Rev. B 1997, 56, R8526-R8529.

63. Tanaka, H.; Kobayashi, A.; Sato, A.; Akutsu, H.; Kobayashi, H. Chemical Control of Electrical Properties and Phase Diagram of a Series of $\lambda$-Type BETS Superconductors, $\lambda$-(BETS) ${ }_{2} \mathrm{GaBr}_{x} \mathrm{Cl}_{4-x}$. J. Am. Chem. Soc. 1999, 121, 760-768. [CrossRef]

64. Mori, H.; Okano, T.; Kamiya, M.; Haemori, M.; Suzuki, H.; Tanaka, S.; Nishio, Y.; Kajita, K.; Moriyama, H. Bandwidth and band filling control in organic conductors. Physica C 2001, 357-360, 103-107. [CrossRef]

65. Kanoda, K. Metal-Insulator Transition in $\mathrm{k}-(\mathrm{ET})_{2} \mathrm{X}$ and (DCNQI) 2 M: Two Contrasting Manifestation of Electron Correlation. J. Phys. Soc. Jpn. 2006, 75, 051007. [CrossRef]

66. Kobayashi, T.; Kawamoto, A. Evidence of antiferromagnetic fluctuation in the unconventional superconductor $\lambda$-(BETS $)_{2} \mathrm{GaCl}_{4}$ by ${ }^{13}$ C NMR. Phys. Rev. B 2017, 96, 125115. [CrossRef]

67. Imajo, S.; Kanda, N.; Yamashita, S.; Akutsu, H.; Nakazawa, Y.; Kumagai, H.; Kobayashi, T.; Kawamoto, A. Thermodynamic Evidence of $d$-Wave Superconductivity of the Organic Superconductor $\lambda$-(BETS $)_{2} \mathrm{GaCl}_{4}$. J. Phys. Soc. Jpn. 2016, 85, 043705. [CrossRef]

68. Sari, D.P.; Asih, R.; Mohm-Tajudin, S.S.; Adam, N.; Hiraki, K.; Ishii, Y.; Takahashi, T.; Nakano, T.; Nozue, Y.; Sulaiman, S.; et al. $\mu S R$ Study of Organic Superconductor $\lambda$-(BETS) ${ }_{2} \mathrm{GaCl}_{4}$. IOP Conf. Ser. Mater. Sci. Eng. 2017, 196, 012047. [CrossRef]

69. Aizawa, H.; Koretsune, T.; Kuroki, K.; Seo, H. Electronic Structure Calculation and Superconductivity in $\lambda$-(BETS) ${ }_{2} \mathrm{GaCl}_{4}$. J. Phys. Soc. Jpn. 2018, 87, 093701. [CrossRef]

70. Imajo, S.; Yamashita, S.; Akutsu, H.; Kumagai, H.; Kobayashi, T.; Kawamoto, A.; Nakazawa, Y. Gap Symmetry of the Organic Superconductor $\lambda$-(BETS) ${ }_{2} \mathrm{GaCl}_{4}$ Determined by Magnetic-Field-Angle-Resolved Heat Capacity. J. Phys. Soc. Jpn. 2019, 88, 023702. [CrossRef] 